\title{
Binocular Vision-Based Augmented Reality System with an Increased Registration Depth Using Dynamic Correction of Feature Positions
}

\author{
Steve Vallerand, Masayuki Kanbara and Naokazu Yokoya \\ Graduate School of Information Science \\ Nara Institute of Science and Technology \\ 8916-5 Takayama, Ikoma, Nara \\ Japan, 630-0101 \\ steve-v@ is.aist-nara.ac.jp
}

\begin{abstract}
In vision-based augmented reality systems, the relationship between the real and virtual worlds needs to be estimated to perform the registration of the virtual objects. This paper suggests a registration method which increases the registration depth of video see-through augmented reality systems using binocular cameras. The method uses both monocular and stereoscopic vision-based techniques in order to perform the registration. Also, the registration method can be combined with a facultative correction of the $2 D$ positions of the feature points. The correction increases the stability and the accuracy of the proposed registration method.
\end{abstract}

\section{Introduction}

Augmented reality (AR) systems enhance user's perception and interaction with the real world. Nowadays, the geometric registration is one of the most important aspects of AR systems. In other words, the real and virtual worlds must be properly aligned to give the illusion that both worlds coexist. Different techniques exist to align the real and the virtual world. In vision-based AR systems, two types of registration techniques exist: monocular and stereoscopic vision-based registration. Monocular registration (MR) uses only one camera. Basically, a MR performs the registration by solving the perspective pose problem from the positions of three or more features in the camera images[1]. On the other hand, stereoscopic registration (SR) uses two cameras and performs the registration from an estimation of the 3D positions of feature points obtained by triangulation[2].

An important characteristic of a registration method is the influence of the distance on the registration quality. A registration method is said to be independent of the dis- tance if the quality of the registration is not influenced when the distance between the camera and the feature points increases. However, if the distance influences the quality of the registration, the registration method is said to be dependant on the distance, and the registration method is consequently limited in registration depth.

Presently, most AR systems use MR since MR is independent of the distance. Although SR is dependent on the distance, stereoscopic computation provides additional information that may help to perform the registration. This paper proposes a new registration method which uses elements of MR and SR to perform the registration. Also, a facultative correction method is presented to increase the registration stability and accuracy of the proposed registration method.

\section{Proposed methods}

\subsection{Registration method}

Our registration method is based on MR to take advantage of the distance independency of this method. We choose to use three points to perform the MR for two principal reasons. First, a registration method using three points is an important aspect of any vision-based system, even for system using MR with four points, since those systems may need to succeed the registration in degenerated conditions, in particular, when one of the four feature points is missing. Secondly, we also want to use stereoscopic computation to perform the registration. Therefore, our registration method is based on MR using three feature points. Then, stereoscopic computation is used to compensate the lack of one feature point.

At first, the 3D positions of three observed points must be retrieved. Determining the $3 \mathrm{D}$ positions of three points from the $2 \mathrm{D}$ positions of those points in an image is called 
the three point space resection problem. Many direct solutions of the three point resection problem have been proposed. Haralick et al., reviewed most of those direct solutions in their paper[3]. The 3D positions of the feature points are retrieved using the Finsterwalder's approach in our system. The multiple solutions obtained are discriminated using stereoscopic projection.

Instead of evaluating all the solutions individually in order to find the best solution, pairs of solutions are evaluated. A pair of solutions (PS) is created with a solution computed from the left image $L$ and a solution computed from the right image $R$. Projection errors $E_{L}$ and $E_{R}$ are computed for each feature point $k$ like illustrated in Figure 1. The global error (GE) of a PS is given by the sum of all the projection errors given by each of the three feature points $(k=1,2,3)$. The PS which gives the smallest error is chosen as the best PS. Then, the left image registration is performed with the left image component of the best PS and the right image registration is performed with the right image component.

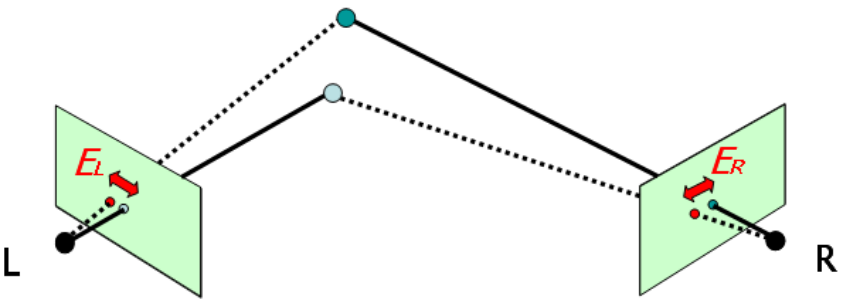

Figure 1. Projection errors for a feature point

\subsection{Correction method}

Instead of performing the registration independently for each image, a facultative correction method to improve the quality of the registration is proposed. The correction method is based on the fact that the 3D positions of corresponding points in binocular images may differ from each image. The difference between the two 3D positions mostly arises from the lack of accuracy when computing the $2 \mathrm{D}$ positions of the feature points. The idea is to correct the 2D positions of the points in the images in order to obtain the same 3D positions for each image.

In other words, we want the GE to equal zero. Corrected 2D positions are computed by applying a rule which tends to diminish the GE. But, the difference between the two 3D positions computed with the corrected points may still be significant. Therefore, the correction process is iterated until the GE is smaller than a predefined threshold value.

\section{Experimental results}

The proposed approach is tested on a desktop PC (SGI visual workstation 330) and a HMD with internal cam- eras (Canon) is used to acquire stereoscopic images. The three corners of a blue triangle marker give the three feature points needed to perform the registration. Colored regions inserted in the triangle allow the system to discriminate between the markers.

The proposed registration method improves the quality of the registration compared to standard SR and standard MR using three points. The correction method influences positively the registration. Once the $2 \mathrm{D}$ positions of the feature points have been corrected, those corrected points can be used to perform a SR with an increased registration depth. All in all, stereoscopic vision applied concurrently with MR improves the registration quality in binocular vision-based AR system using three feature points based registration. Figure 2 shows some registration images obtained with the proposed registration and correction methods.
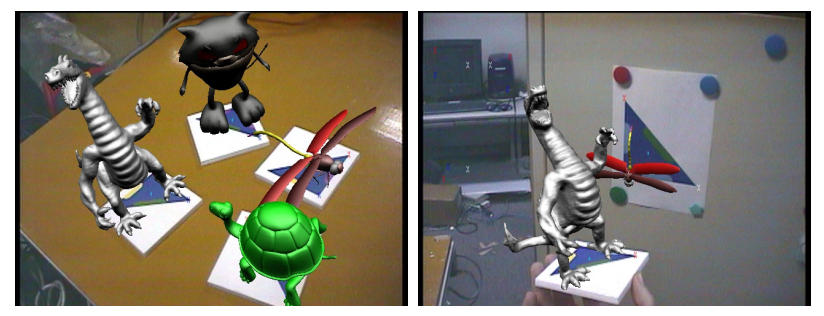

Figure 2. Examples of registration images.

\section{Conclusion}

This paper has proposed a new method for performing the registration in a binocular vision-based AR system. The proposed registration method is independent of distance even if only three feature points are used to perform the registration. Also, the correction method increases the registration depth of SR systems. In future work, we want to evaluate the effect of the correction method in a system using MR with four feature points.

\section{References}

[1] T. Okuma, K. Kiyokawa, H. Takemura and N. Yokoya, "An Augmented Reality System Using a Real-time Vision Based Registration", ICPR 98: Proceedings 14th International Conference on Pattern Recognition, Vol. 2, pp. 1226-1229, 1998.

[2] M. Kanbara, T. Okuma, H. Takemura and N. Yokoya, "A Stereoscopic Video See-through Augmented Reality System Based on Real-time Vision-based Registration", Proc. IEEE Virtual Reality 2000, pp. 255-262, 2000.

[3] R.M. Haralick, C.-N. Lee and K. Ottenberg, "Analysis and Solutions of The Three Point Perspective Pose Estimation Problem”, Proc. CVPR '91, pp. 592-598, June 1991. 